\title{
Les modèles mathématiques en épidémiologie. L'exécution du testament de Sir Ronald Ross
}

\section{DAVID BEKOLLE}

Membre de l'Académie, professeur de Mathématiques à l'Université de Yaoundé I, vice-recteur honoraire à l'Université de Ngaoundéré, Département de Mathématiques et Informatique, Faculté des Sciences, Université de Ngaoundéré, B.P. 454, Ngaoundéré, Cameroun.

Courriels: dbekolle@,univ-ndere.cm \& dbekolle@gmail.com

\section{Résumé}

Cette article est une évocation personnelle des différentes phases de l'implantation au Cameroun et en Afrique Centrale du thème de recherche "Epidémiologie Mathématique". Il analyse également les enjeux et les perspectives de ce thème dans une portée pluridisciplinaire.

\begin{abstract}
.
This article is the author's testimony to the successive stages of the setting in Cameroon and Central Africa of the research theme "Mathematical Epidemiology". It also analyses the challenges and prospects of this theme from a multidisciplinary point of view.
\end{abstract}

Received: 11/02/2019

Accepted: 04/08/2019

DOI: https//dx.doi.org/10.4314/jcas.v15i2.1

(C) The Authors. This work is licensed under the Creative Commons Attribution 4.0 International Licence. 


\section{Introduction}

Sir Ronald Ross (1857-1932) était un médecin bactériologiste et entomologiste britannique de l'Armée des Indes. Il montra en 1897 que la transmission du paludisme se fait par un moustique. En 1902, il reçut le prix Nobel de médecine pour ses travaux sur le paludisme. Il a construit le premier modèle mathématique du paludisme, qu'il a publié dans son livre “The prevention of malaria" [5]. Grâce à son modèle, il a démontré que pour éradiquer le paludisme, il n'est pas nécessaire d'éliminer toute la population des moustiques, mais il suffit de la ramener endessous d'un certain 'seuil' dont il donnait l'expression mathématique en fonction des paramètres. Il infligeait ainsi une réponse cinglante à ses contradicteurs qui objectaient que le paludisme allait persister tant qu'il y aurait une population d'anophèles.

Cette réponse de Sir Ronald Ross, qui figure dans son livre, sert de testament aux mathématiciens camerounais, et leur a instruit un thème de recherche, l'Epidémiologie Mathématique. En voici les termes exacts:

"The mathematical treatment adopted in section 28 has been met with some questioning by critics. Some have approved of it, but others think that it is scarcely feasible owing to the large number of variables which must be considered. As a matter of fact, all epidemiology, concerned as it is with the variation of disease from time to time or from place to place, must be considered mathematically, however many variables are implicated, if it is to be considered at all. To say that a disease depends upon certain factors is not to say much, until we can also form an estimate as to how largely each factor influences the whole result. And the mathematical method of treatment is really nothing but the application of careful reasoning of the problem at issue."

Voici la traduction en français de cette citation" "Le traitement mathématique adopté à la section 28 a fait l'objet d'interrogations de la part de critiques. Certains l'ont approuvé, mais d'autres pensent qu'il est à peine réalisable, à cause du grand nombre de variables à considérer. En fait, toute l'épidémiologie, concernée comme elle est par la variation de la maladie d'un instant à un autre ou d'un lieu à l'autre, doit être considérée mathématiquement, nonobstant la quantité de variables impliquées, si elle (l'épidémiologie, $\mathrm{NDT}^{2}$ ) doit être un tant soit peu prise en considération. Dire qu'une maladie dépend de certains facteurs ne veut pas dire grand chose, tant qu'on ne formule pas aussi une estimation quantitative de l'influence de chaque facteur sur le résultat définitif. Et la méthode mathématique du traitement n'est en fait rien d'autre que l'application de raisonnements approfondis au problème en question."

L'exécution du testament de Sir Ronald Ross s'est effectuée pendant 20 ans au Cameroun et en Afrique Centrale. Cet article relate différentes phases de l'implantation dans la sous-région du thème de recherche "Epidémiologie Mathématique". Partant de sa genèse, la première partie (sections 2-5) en évoque les étapes préliminaires: l'école fondatrice, la préparation des premières thèses de doctorat, les premiers ateliers et l'instauration du réseau sous-régional EpiMath $^{3}$. La deuxième partie (sections 6-10) présente des étapes opérationnelles et structurantes de la formation par la recherche: la mise en place du premier Master entièrement dédié à ce thème, la préparation et les soutenances au Cameroun des premières thèses de Doctorat/ PhD. La conclusion (section 11) est une exhortation à une collaboration entre mathématiciens, biologistes et professeurs de

${ }^{1}$ Traduttore, traditore! Traduire, c'est trahir!

${ }^{2}$ Note du traducteur.

${ }^{3}$ Réseau d'Afrique Centrale de Modelisation Informatique et Mathématique en Epidémiologie et Immunologie. 
médecine dans la recherche en épidémiologie. Enfin, à la section 12, nous esquissons les perspectives d'une telle collaboration.

Une collaboration à cette échelle est inédite au Cameroun et en Afrique Centrale; il faudra donc surmonter les obstacles inhérents à sa réalisation. Avec l'assurance de leur disponibilité, les mathématiciens mettent à disposition leur expertise dans les domaines suivants:

1. Elaboration et analyse mathématique des modèles épidémiologiques;

2. Simulation informatique des modèles et confrontation aux données du terrain;

3. Détermination des seuils, contrôle optimal des interventions et de leurs coûts.

Au-delà des problématiques de recherche, ces trois domaines relèvent d'un véritable "génie mathématique" ("mathematical engineering"). Pour étayer cette exhortation à une recherche pluridisciplinaire, deux annexes donnent une liste des mathématiciens seniors permanents impliqués (Annexe 1) et une sélection de dix de leurs publications (Annexe 2), à laquelle il convient d'adjoindre deux publications majeures [3, 4] citées dans la bibliographie.

Dans tout le texte, la personne qui est désignée comme premier collègue de l'Université de Yaoundé I est l'auteur du présent article. Le deuxième collègue de l'Université de Yaoundé I l'ordre de céans n'est pas régi par la préséance est le doyen Bitjong Ndombol.

\section{La Genèse du programme}

Trieste, Italie, été 1998. Deux collègues de l'Université de Yaoundé I effectuaient un séjour de recherche à l'ICTP (International Centre for Theoretical Physics). Ils étaient issus de l'école française de Mathématiques dites 'Pures'
(Topologie Algébrique, Analyse Classique) et ils allaient réaliser pendant ce séjour les travaux de recherche les plus importants de leur carrière. L'année précédente, ils avaient participé à Djéregbé (Bénin) à l'Ecole du CIMPA 4 “Théorie du Contrôle", Durant laquelle pendant trois semaines, d'éminents conférenciers leur avaient exposé différents aspects de la Théorie du Contrôle, ainsi que leurs applications. Ils avaient alors compris que pour fabriquer une aile d'avion, on n'allait pas directement tailler dans l'acier ou dans le matériau idoine. La conception relevait d'abord de la théorie mathématique du Contrôle des Equations aux Dérivées Partielles ${ }^{5}$, qui contribuait à élaborer un modèle d'aile d'avion. On réalisait ensuite une expérimentation 'in silicio', une simulation informatique pour s'assurer des qualités du modèle, en vue de sa validation.

A la suite de cette école, il avait été institué à l'IMSP (Institut de Mathématiques et Sciences Physiques) de Porto Novo, une formation de troisième cycle de Théorie du Contrôle. Y avaient été admis deux jeunes camerounais: Jean Claude Kamgang, titulaire d'un DEA de Géométrie de l'Université de Paris VI, et Samuel Bowong, titulaire d'une Maitrise de Mécanique de l'Université de Dschang. Ces deux étudiants seront reçus au DEA et admis en doctorat en Contrôle et Automatique sous la direction du professeur Gauthier Sallet (INRIA ${ }^{6}$ et Université de Metz), directeur de la formation.

Mathématiciens africains, exerçant en Afrique, l'un d'eux assumant déjà des responsabilités académiques (chef de département), les deux collègues de l'Université de Yaoundé I avaient convenu rapidement pendant leur séjour à Trieste de l'impérieuse nécessité d'implanter un thème de recherche en Mathématiques comportant des applications aux résolutions de problèmes de

4Centre International de Mathématiques Pures et Appliquées, Nice (France). Le directeur en 'était Claude Lobry. 5Conférenciers pour le cours de Contrôle des Equations aux Dérivées Partielles: Nicolas Burq et Brice Allibert. 6Institut National (français) de Recherche en Informatique et Automatique. 
développement. Pour en déterminer un thème, ils avaient effectué des recherches sur internet, la pertinence et la consistance mathématique du thème étant les critères majeurs de son adoption. L'autre collègue est revenu à un moment avec le thème suivant qui a emporté leur adhésion commune: "Mathématiques et Malaria"s.

Ils n'y connaissaient rien l'un et l'autre. Sans délai, ils se sont attelés à l'organisation d'une école scientifique, pluridisciplinaire et internationale sur le thème Mathématiques et Malaria". Ils avaient déjà l'assurance de l'organisation à Yaoundé de l'Ecole du CIMPA “Outils Informatiques dans la Recherche en Systèmes Dynamiques Complexes" 9. Pour cette nouvelle école, il fallait trouver des conférenciers. Ils en ont contacté deux avec succès sur internet: Ingemar Nåsell (KTH, Stockholm), Guenther Hasibeder (Vienne).

Ils ont ensuite demandé à Gauthier Sallet de coopérer à ce projet, vu sa connexité avec la Théorie du Contrôle et l'Automatique; la vitesse avec laquelle il va s'imprégner du sujet devrait servir d'exemple, en même temps qu'il infléchira les projets de thèse de Jean Claude Kamgang et Samuel Bowong vers des problèmes de modélisation mathématique en Epidémiologie. Par son canal, l'autre collègue de l'Université de Yaoundé I gagnera la collaboration de Martin Eichner (Tübingen), biostatisticien, qui sera également conférencier de cette école fondatrice.

Ils allaient aussi obtenir le soutien déterminant du professeur Albert Samè Ekobo, spécialiste de Parasitologie à la Faculté de Médecine et des Sciences Biomédicale de l'Université de Yaoundé
$\mathrm{I}^{10}$. La 'french connection' leur assurera la participation comme conférenciers des deux chercheurs suivants de l'IRD ${ }^{11}$ :

1. Didier Fontenille, entomologiste;

2. Gaston Pichon, parasitologue.

Surtout, ils bénéficieront de l'adhésion et du soutien scientifique du Professeur Christophe Rogier de l'Institut de Médecine Tropicale du Service de Santé des Armées (Marseille). Médecin militaire (médecin-colonel, aujourd'hui médecin général adjoint), il était alors responsable de la gestion des épidémies de paludisme dans les bases militaires françaises d'Afrique. Professeur agrégé de médecine, il est spécialiste de biologie moléculaire. Il les a rassurés dès le début, quant à la pertinence du thème de recherche "Mathématiques et Malaria". La recherche médicale est limitée parce qu'en vertu des lois de la bioéthique, elle n'a pas le droit d'effectuer des expériences sur des humains au-delà d'une période de six mois. On procède ensuite à l'analyse statistique des données collectées. Mais ces données s'avèrent insuffisantes dans plusieurs cas (voir ci-dessous la section consacrée aux données de Dielmo et Ndiop). Peut-on ensuite extrapoler les résultats obtenus et accéder à une connaissance de l'évolution ultérieure des phénomènes observés pendant six mois? Dans la configuration actuelle des sciences, les solutions doivent être données par les mathématiciens, dans une démarche de collaboration avec les autres disciplines (biologie, médecine, ...); il s'agit d'élaborer des modèles mathématiques qui donnent lieu à des problèmes d'évolution, dont la variable principale est le temps (l'instant). Au-

\footnotetext{
7'Malaria' est la traduction anglaise du mot 'paludisme'. Néanmoins, ce mot anglais est utilisé dans la littérature scientifique francophone.

${ }^{8}$ Rétablissons la vérité historique. La discipline des Biomathématiques a été présentée pour la première fois à la communauté mathématique camerounaise et d'Afrique Centrale en 1997 lors d'un séminaire de l'Union Mathématique Africaine tenu à l'Université de Yaoundé I. Les auteurs de cette présentation étaient deux jeunes scientifiques de l’Université de Buea: Gideon Akumah Ngwa et William Shu. Ils ont publié en 2000 un article [4] sur la modélisation de la transmission du paludisme qui a nourri l'apprentissage de tous les doctorants camerounais de la discipline. ${ }^{9}$ Ecole Normale Supérieure, Université de Yaoundé I. Conférenciers pléniers: Adrien Douady, Pierrette Sentenac, Michel Zinsmeister, Robert Roussarie, Albert Cohen, Hamidou Touré.

${ }^{10}$ Bien sûr, dès le départ, il fallait susciter l'adhésion et la collaboration des professeurs de médicine.

${ }^{11}$ Institut (français) de Recherche pour le Développement
} 
delà des statistiques, les théories mathématiques utilisées sont:

1. Les équations différentielles, les équations aux dérivées partielles;

2. Les systèmes dynamiques en dimension finie et en dimension infinie;

3. La théorie des probabilités et les équations différentielles stochastiques;

4. La théorie du contrôle (optimal, géométrique,...) et l'automatique.

Cette liste n'est pas exhaustive, les méthodes peuvent emprunter à toutes les branches des mathématiques, ce qui explique l'intérêt de la modélisation mathématique dans le développement de la science mathématique ellemême.

\section{An 2000: Célébration de l'Année Mathématique Mondiale au Cameroun}

En l'an 2000, en plus de la tutelle du Ministère de l'Enseignement Supérieur, les mathématiciens camerounais ont joui de la tutelle du Ministère de la Recherche Scientifique et Technique. La base de cette tutelle était la qualité de mathématicien de renommée internationale du ministre Henri Hogbé Nlend.

Sous la tutelle du Ministère de la Recherche Scientifique et Technique, l'Année Mathématique Mondiale 2000 a été célébrée au Cameroun dans la magnifique salle de conférences de ce ministère dans la période allant du 28 août au 15 septembre 2000. Le CIMPA était co-organisateur de cette manifestation scientifique. La célébration comportait deux articulations:

1. 28 août-02 septembre 2000: Ecole Internationale "Statistiques et Agronomie";

2. 04-15 septembre 2000: Ecole Internationale "Mathématiques et Malaria".

L'Ecole Internationale "Statistiques et Agronomie" avait pour conférenciers pléniers, deux statisticiens de l'INRA (Institut National de Recherche Agronomique) d'Avignon, Claude Bruchou et Jacques Badia. L'auditoire eut droit à un excellent cours d'Analyse des Données ${ }^{12}$, illustré par des exemples pratiques, de la part de 'statisticiens qui savent sélectionner des semences'.

L'Ecole Internationale "Mathématiques et Malaria" avait été très animée en raison de la multiplicité des disciplines présentes dans son équipe de conférenciers (mathématiciens, biologistes, entomologistes, parasitologues, biostatisticiens, professeurs de médecine, .... $)^{13 ; 14}$. Les échanges avaient été vifs autour de deux points de vue antagonistes:

- Elaborer des modèles mathématiques de transmission de la malaria, à la suite de Sir Ronald Ross et de ses successeurs;

- Elaborer des modèles mathématiques et informatiques pour décrire et comprendre des phénomènes plus circonscrits (résistance, lutte anti-vectorielle,...).

Le premier point de vue a été rejeté par la direction scientifique de l'Ecole ${ }^{15}$. La transmission de la malaria sera toujours active en Afrique

\footnotetext{
L'Analyse des Données est une discipline sérieuse qu'il convient d'apprendre auprès de spécialistes. Rappel à l'ordre à tous les chercheurs qui prétendent l'utiliser et dont les résultats se réduisent souvent à la statistique descriptive élémentaire! Les responsables académiques qui invitent les professeurs d'Analyse des Données à animer de simples séminaires devraient les inviter pour de longues périodes à dispenser de véritables cours.

${ }^{13}$ L'Ecole a aussi bénéficié d'une intervention lumineuse du professeur Rose Leke. Il faut mentionner également la participation assidue du professeur Charles Félix Bilong Bilong, du Département de Biologie et Physiologie Animales de la Faculté des Sciences de l’Université de Yaoundé I.

${ }^{14}$ Autre conférencier de marque: le médecin-général Jean Dutertre. Dans le cadre de l’OCEAC (Organisation de Coordination pour la Lutte contre les Endémies en Afrique Centrale), sur les pas des médecins-colonels Eugène Jamot et Pierre Richet, il avait, dans les années soixante, dirigé des équipes de lutte contre la maladie du sommeil et le paludisme au Cameroun. Jean Dutertre est aussi l'auteur d'un modèle mathématique du paludisme [1]. Pour sa participation à la célébration de l’Année Mondiale des Mathématiques au Cameroun, se référer à son article [2]

${ }^{15} \mathrm{Ce}$ rejet n'a heureusement pas été radical. Dans ce sens, S.Y. Tchoumi a soutenu en 2010 à l'Université de Ngaoundéré un mémoire de Master intitulé "Prise en compte de la variable spatiale dans la modélisation de la moustiquaire imprégnée". Cf. également l’article de J.C. Kamgang,V.C. Kamla et S.Y. Tchoumi [3] qui présente une étude plus extensive de l'impact de l’utilisation de la moustiquaire imprégnée.
} 
subsaharienne, le moustique étant dans un environnement favorable. Si au sud de la France, les anophèles ont été éliminés à la fin du XIXème siècle grâce à l'assèchement des marais, il n'est pas possible de faire de même dans notre région sans porter atteinte aux écosystèmes et par suite, à l'environnement de la planète ${ }^{16}$. Par contre, l'adoption du deuxième point de vue assignait aux mathématiciens la mission d'apporter une contribution scientifique aux mécanismes d'aide à la décision. Ils allaient faire "œuvre utile", en plaçant les mathématiques et les mathématiciens dans une position de choix dans la mission d'appui au développement.

\section{Les données de Dielmo et Ndiop}

Dielmo et Ndiop sont deux petits villages du Sénégal, présentant des profils environnementaux différents (pluviométrie, température, altitude,... $)^{17}$. Ils sont distants de $5 \mathrm{~km}$ et situés près de la frontière gambienne. Dielmo présente la caractéristique d'un paludisme holo-endémique, alors que Ndiop présente la caractéristique d'un paludisme méso-endémique.

Trois structures scientifiques et techniques (IRD, Institut Pasteur de Dakar, Ministère (sénégalais) de la Santé et de la Prévention Médicale) ont été réunies pour étudier le paludisme dans ces deux villages pilotes. Elles y ont installé deux stations d'études avec le consentement des villageois euxmêmes. "A partir de l'histoire individuelle biologique, clinique et épidémiologique de 700 villageois suivis quotidiennement, il s'agit de décrire l'hétérogénéité individuelle de la réponse à l'infection et à la maladie, de rechercher ses déterminants biologiques et épidémiologiques innés et acquis, et de quantifier leur importance" ${ }^{18}$.

La plateforme a été déployée pour la première fois en 1989. Les villageois ont été médicalement suivis, systématiquement diagnostiqués et soignés $\mathrm{du}$ paludisme pendant six mois consécutifs. Au bout de la période, une analyse statistique des données collectées a été effectuée.

Quelles étaient les problématiques de ces équipes de recherche? Quels étaient les résultats escomptés? L’opération a été renouvelée à plusieurs reprises. Les problématiques relevaient de la compréhension du fonctionnement du système immunitaire de l'homme par rapport au parasite, le plasmodium. Elles renvoyaient plus loin à la conception d'un vaccin efficace en zone endémique - en particulier, dans notre sous-région - et à l'explication des échecs des essais précédents. Il est apparu que le temps imparti par les lois de la bioéthique était trop court pour parvenir à leurs fins.

\section{Les premiers ateliers "Mathématiques et Malaria". L'extension à d'autres maladies et l'instauration du Réseau EpiMath.}

Il fallait ensuite lancer le programme de recherche sur le thème "Mathématiques et Malaria". Cela devait commencer par l'instauration d'un programme de formation par la recherche (Master et Doctorat).

\footnotetext{
${ }^{16}$ Le lecteur intéressé pourra consulter les conclusions du Projet Garki (directeur: Louis Moulineaux), http://garkiproject.nd.edu. Ce projet a été initié par l'OMS et le gouvernement nigérian qui ont dépensé plus de 6 millions de dollars US pour essayer d'éradiquer le paludisme dans 164 villages du nord du Nigéria. Selon Joseph Faye (Institut Pasteur, Dakar), "le modèle mathématique simulant assez fidèlement l'épidémie de P. falcifarum peut calculer les conséquences parasitologiques de modifications déterminées de la capacité vectorielle, du traitement médicamenteux de masse". Il décrit ensuite les conséquences à long terme sur la lutte antipaludique: "Dans l'avenir, les pulvérisations à effet rémanent et les administrations massives de médicaments ne doivent pas être recommandées comme méthode de lutte antipaludique dans les zones rurales de savane soudanienne d'Afrique". Cf. http://www.slideshare.net/atelier-paludisme/faye-s4.

${ }^{17}$ En 2019, Ezékiel Dangbé soutiendra à l’Université de Ngaoundéré une thèse de Doctorat/Ph.D intitulée "Impact des variations environnementales sur la transmission des maladies infectieuses: cas du choléra et du paludisme".

${ }^{18} \mathrm{http}: / /$ www.senegal.ird.fr/l-ird-au-senegal/sites-principaux/les-stations-de-dielmo-et-ndiop. Christophe Rogier est l'un des directeurs de ce programme.
} 
Le 13 avril 2000, le premier collègue de l'Université de Yaoundé I a été nommé chef de département de Mathématiques de la Faculté des Sciences de l'Université de Yaoundé I. Les systèmes d'équations du paludisme étant de grande taille et non-linéaires, les mathématiques pouvaient en donner des théorèmes d'existence et d'unicité des solutions. Mais elles n'en donnaient pas des expressions explicites. Il faut recourir à l'Analyse Numérique pour en donner des valeurs approchées et dans ce cas, on utilise l'outil informatique. Le même outil est utilisé pour effectuer des simulations numériques, qui donnent des courbes d'évolution, des surfaces et des volumes qui permettent de visualiser l'évolution du système.

Malheureusement, à la Faculté des Sciences de l'Université de Yaoundé I, le département d'Informatique, bien que contigu au département de Mathématiques, en est séparé par une grille métallique. De plus, les organisations des enseignements dans les deux départements sont totalement disjointes. Les unités d'enseignement d'Informatique sont dispensées aux étudiants de Mathématiques par des mathématiciens; comme les unités de Physique, elles sont devenues des U.E. pour la forme, à valider et à oublier, leur exécution manque de sérieux. Bref, il a été impossible au chef de département de Mathématiques de mettre en place un Master d'Ingénierie Mathématique, qu'il appelait de ses vœux.

Pour implanter le thème de recherche "Epidémiologie Mathématique", il avait besoin de faire assurer les enseignements suivants:

1. Epidémiologie Biologique et Clinique;

2. Modèles hôtes-parasites;

3. Modèles Probabilistes;
4. Statistiques et Analyse des Données;

5. Génie Logiciel;

6. Ateliers de programmation;

7. Analyse Fonctionnelle et Théorie des Distributions;

8. Analyse Numérique;

9. Equations aux Dérivées Partielles;

10. Systèmes Dynamiques.

Le 22 septembre 2003 et le 27 novembre 2003, Jean Claude Kamgang et Samuel Bowong Tsakou soutenaient respectivement à l'Université de Metz des thèses de Doctorat intitulées:

- "Contribution à la stabilisation des systèmes mécaniques: contribution à l'étude de la stabilité des modèles épidémiologiques";

- "Contribution à la stabilisation et stabilité des systèmes non linéaires: Application à des systèmes mécaniques et épidémiologiques".

Deux autres doctorants avaient entamé leurs travaux de thèse sous la direction de Gauthier Sallet: Jean Jules Tewa et Joseph Mbang. Ils soutiendront respectivement à l'Université de Metz le 26 juillet 2007 et le 20 mai 2009 leurs thèses de doctorat intitulées:

- "Analyse globale des modèles épidémiologiques multicompartimentaux: application à des modèles intra-hôtes du paludisme et du V.I.H";

- "Modèles intra-hôtes avec retard: application à des modèles intra-hôtes du paludisme et du V.I.H$1 ”$.

Pour remédier aux problèmes d'environnement scientifique, deux ateliers scientifiques "Mathématiques et Malaria" ont été organisés à Yaoundé du 25 au 29 août 2003 et à Brazzaville ${ }^{19}$ du 12 au 23 juillet 2004. La tenue de ces ateliers a bénéficié du soutien financier de l'Agence Universitaire de la Francophonie (AUF, Projet PAS

${ }^{19}$ Président du comité d'organisation: Jean Luc Dimi, Université Marien Ngouabi. Le réseau EpiMath va établir un tandem Cameroun-Congo. 
01), dont le bureau Afrique Centrale de Yaoundé a abrité les travaux du premier atelier ${ }^{20}$.

A l'atelier de Yaoundé, les trois (3) problèmes suivants ont été dûment identifiés:

1. l'impact à long terme de l'utilisation des moustiquaires imprégnées dans la lutte contre le paludisme;

2. les déterminants écologiques et anthropologiques de la dynamique des populations de plasmodiums; ce problème sera notamment étudié en liaison avec le problème de résistance aux antipaludiques;

3. les caractéristiques de l'immunité antipaludique, acquise ou innée, observée chez les populations vivant en zone d'endémie; il s'agit de caractéristiques à prendre en compte pour la compréhension et la modélisation du paludisme.

L'atelier de Brazzaville comportera des cours sur le thème "Applications à des maladies tropicales émergentes et ré-émergentes". En raison de la similitude des méthodes de modélisation, il y fut décidé d'étendre les investigations à d'autres maladies tropicales: tuberculose, fièvre Ebola, trypanosomiase, lèpre, leishmaniose, fièvre jaune,... et au V.I.H. Martin Eichner présenta dans cet atelier un simulateur d'épidémie de grippe, qu'il avait mis au point avec Markus Schwehm, spécialiste d'Analyse Numérique, également présent. C'était un outil d'aide à la décision, construit à base de biostatistiques, de probabilités, d'analyse numérique, et bien sûr d'informatique (modélisation individu-centrée et modélisation à base d'agents).

Lors de cet atelier, Paul Boumandouki, épidémiologiste congolais, a présenté les données statistiques de la dernière épidémie d'Ebola qui venait d'éclater dans le Nord du pays. Martin
Eichner passa le même après-midi torride à vérifier la complétude des données. Trois ans plus tard, il reviendra avec Markus Schwehm exposer un simulateur de la fièvre Ebola. En cas de nécessité d'une intervention médicale ou de dispositions particulières (quarantaine, mesures d'hygiène,...) le simulateur se colorie en rouge sang!

Cet atelier a aussi le théâtre de l'instauration et de l'adoption des statuts du Réseau EpiMath "Réseau d'Afrique Centrale de Modélisation Informatique et Math ématique en Epidémiologie et en Immunologie". Le réseau a coopté dès sa création le Docteur Carole Eboumbou, jeune biologiste, bientôt recrutée comme enseignante à la Faculté de Médecine et des Sciences Pharmaceutiques de Douala. C'est sous la bannière du Réseau EpiMath que seront organisés les futurs ateliers (Yaoundé, 7-11 novembre 2005, Ngaoundéré, 06-11 Septembre 2008, 16-18 Janvier 2012 21, 17-19 Septembre 2017; Brazzaville, 5-10 mars 2007, 6-12 septembre 2009 et 5-9 mai 2014).

L'atelier EpiMath I, tenu à Yaoundé en novembre 2005, bénéficiera d'une participation appuyée de l'IRD (Unité de Recherche GEODES), avec les conférenciers suivants:

1. Pierre Auger, membre de l'Académie Française des Sciences;

2. Nicolas Bacaer ${ }^{22}$;

3. Hassan H'bid et Mohammed Khaladi, de l'Université de Marrakech.

Les autres exposés ont été donnés par Christophe Rogier, Gauthier Sallet, Abderrahman Iggidr (INRIA et Université de Metz), Martin Eichner, G. Akumah Ngwa, Jean Claude Kamgang (Ngaoundéré), Alphonse Kalala (Kinshasa),

${ }^{20} \mathrm{Il}$ est remarquable que l'Agence Universitaire de la Francophonie ait accordé son soutien au projet à ses débuts, malgré (ou peut-être à cause de) son caractère novateur. Son soutien restera constant tout au long du parcours.

${ }^{21}$ Conférencier invité: Adamou Otto (Université Abdou Moumouni, Niamey). Cet expert étudie des modèles épidémiologiques en utilisant des méthodes de calcul formel, notamment les bases de Groebner.

${ }^{22}$ Ancien élève de l'Ecole Normale Supérieure de Paris, il présenta un système (d'équations aux dérivées partielles) de réaction-diffusion modélisant la propagation de la résistance à un médicament antipaludique. Il exposa également un modèle de la leishmaniose. 
Aminou Layaka (N'Djamena) et Wilson SamaTitanji2 ${ }^{23}$ (Swiss Tropical Institute, Bâle (Suisse)).

\section{Octobre 2008: Le premier Master d'Epidémiologie Mathématique s'ouvre à l'Université de Ngaoundéré}

Le 10 septembre 2005, le premier collègue de l'Université de Yaoundé I a été nommé vicerecteur à l'Université de Ngaoundéré. Immédiatement après, le 14 novembre de la même année, il a été nommé, cumulativement avec ses fonctions de vice-recteur, chef de département de Mathématiques et Informatique à la Faculté des Sciences de la même institution. Comparé à l'Université de Yaoundé I, l'effectif des membres du département est plus modeste avec une dizaine de membres, équitablement répartis entre Mathématiques et Informatique. Ils sont jeunes et ambitieux, assistants et chargés de cours. La veille de son installation comme vice-recteur, ils l'accueillent chaleureusement en sa qualité de professeur, promis à établir les bases d'un développement du département, qui leur permettra d'évoluer dans leurs carrières.

A Ngaoundéré, il est chef d'un département à la fois de Mathématiques et d'Informatique. Ce département coordonne une seule filière de Licence dénommée "Sciences et Techniques de l'Informatique (STI)", avec un tronc commun au premier niveau et deux options aux niveaux II et III:

- Architecture et Réseaux;

- Calcul Scientifique.

Hommage au Recteur Maurice Tchuenté24, si la deuxième option était davantage orientée vers les mathématiques et la première vers l'informatique, à la fois mathématique et informatique ${ }^{25}$. Contrairement à un point de vue répandu dans les sphères scientifiques, c'est cette organisation des enseignements qui est conforme à l'histoire et à la philosophie des sciences ${ }^{26}$.

L'effectif des étudiants est raisonnable, quatrevingts au maximum au premier niveau, et cinquante au maximum au niveau III. Le cadre est propice au travail, les enseignants sont dévoués.

Il fallait dans un premier temps initier un cycle de Master. En raison des effectifs des étudiants, on ne pouvait proposer que deux parcours: un pour les étudiants issus de l'option Calcul Scientifique, l'autre pour les étudiants issus de l'option Architecture et Réseaux. L'environnement étant maintenant favorable, il a été décidé de dénommer le master "Master d'Ingénierie Mathématique et Informatique" et de le munir de deux parcours:

1. Epidémiologie Mathématique;

2. Systèmes et Logiciels en Environnements Distribués.

En prélude à l'ouverture du Master, il a été organisé à l'Université de Ngaoundéré l'Atelier EpiMath III dans la semaine allant du 6 au 11 septembre 2008. Y ont participé les conférenciers suivants, qui ont présenté des exposés de recherche sur des sujets à établir dans le Master:

1. Reinhard Vonthein (Lübeck);

2. Markus Schwehm (Tübingen);

3. Gauthier Sallet (INRIA et Université de Metz);

4. Dominique Bicout (Ecole Vétérinaire, Lyon);

5. Pape Ibrahima Ndiaye (Bambeye, Sénégal);

6. Jean Luc Dimi (Marien Ngouabi, Brazzaville);

7. Macaire Batchi (Marien Ngouabi, Brazzaville); tous les étudiants devaient acquérir une formation

${ }^{23}$ Alors doctorant, ce détenteur d'un Master de l'Université de Buéa a soutenu à l'Université de Bâle en 2006 une thèse de PhD intitulée "Statistical Analysis of Within-host Dynamics of Plasmodium Falcifarum infections", préparée sous la direction de Tom Smith. Il a présenté une étude sur la durée de l'infection à Plasmodium falciparum.

${ }^{24}$ Informaticien de tout premier rang, il a été recteur à l'Université de Ngaoundéré de 1998 à 2000. Comme auparavant à Dschang, ensuite à Douala, il a marqué de son empreinte l'organisation du département de Mathématiques et Informatique de la Faculté des Sciences.

${ }^{25} \mathrm{~A}$ l'époque, des étudiants de l'option Calcul Scientifique tenaient des cyber-cafés dans le quartier universitaire de Dang.

${ }^{26} \mathrm{Il}$ est urgent qu'un comité formé de philosophes des Sciences soit institué à l'effet d'analyser l'organisation des enseignements dans l'université camerounaise. 
8. Carole Eboumbou (Faculté deMédecine et des Sciences Biomédicales, Yaoundé I);

9. Hervé Bogreau (Institut de Médecine Tropicale du Service de Santé des Armées, Marseille).

Reinhard Vonthein est un statisticien spécialiste de l'Analyse des Données. Il se sert avec une dextérité incroyable du logiciel R. Dans son université, il est au point de départ de chaque opération de collecte des données; c'est dans son service qu'on arrête le protocole expérimental ${ }^{27}$.

A l'occasion de cet Atelier EpiMath, Gauthier Sallet, Dominique Bicout et Pape Ibrahima Ndiaye ont dispensé les premiers cours du Master.

\section{Les enseignements du Master}

Grâce à une subvention de l'Agence Universitaire de la Francophonie (AUF, Projet MIME ${ }^{28}$ ), le département de Mathématiques et Informatique de la Faculté des Sciences de l'Université de Ngaoundéré a invité pendant deux ans des missionnaires chevronnés à dispenser les enseignements suivants du Master d'Epidémiologie Mathématique (Tableau 1):

Tableau 1. Liste des missionnaires du Master.

\begin{tabular}{|l|l|}
\hline Intitulé de l'UE & Noms des enseignants \\
\hline $\begin{array}{l}\text { Epidémiologie Biologique } \\
\text { et Clinique }\end{array}$ & C. Eboumbou \\
\hline Modèles hôtes-parasites & G. Sallet, D. Bicout, J.J. Tewa (ENSP, UYI) \\
\hline Systèmes Dynamiques & Boulchard Mewoli (FS, UYI) \\
\hline $\begin{array}{l}\text { Statistiques et Analyse des } \\
\text { Données }\end{array}$ & Michel Ndoumbè Nkeng (IRAD) \\
\hline $\begin{array}{l}\text { Calculabilité et Complexité } \\
\text { des Algorithmes }\end{array}$ & Basile Louka (Informatique, FS, UYI) \\
\hline
\end{tabular}

Le contenu de l'UE "Epidémiologie Biologique et Clinique" a été mis au point à l'Institut de Médecine Tropicale du Service de Santé des Armées (Marseille) sous la direction du professeur Christophe Rogier. Carole Eboumbou et Hervé Bogreau faisaient de l'équipe de rédaction.

Le responsable de l'UE Statistiques et Analyse des Données, Michel Ndoumbè Nkeng, est ${ }^{27}$ Communication de Dominique Bicout: "les opérations de collecte de données sont très coûteuses".

28"Modélisation Informatique et Mathématique en Epidémiologie".

${ }^{29}$ Le Master d’IngénierieMathématique et Informatique de la Faculté des Sciences de l’Université de Ngaoundéré est l'affaire des enseignants de Mathématiques et Informatique et de tous les établissements scientifiques et technologiques de l’Université de Ngaoundéré (Faculté des Sciences, IUT, ENSAI) ingénieur agronome, docteur en statistiques, alors chef de service de Biométrie à l'IRAD (Institut de Recherche Agronomique pour le Développement). Un autre 'statisticien qui sait sélectionner des semences'.

Les autres cours du Master ont été assurés par des enseignants permanents ${ }^{29}$ (Tableau 2):

Tableau 2. Liste des enseignants permanents affectés au Master.

\begin{tabular}{|l|l|} 
Intitulé de l'UE & Noms des enseignants \\
\hline Analyse Fonctionnelle & D.E. Houpa Danga (FS) \\
\hline $\begin{array}{l}\text { Distributions et Equations } \\
\text { aux Dérivées Partielles }\end{array}$ & D.E. Houpa Danga (FS) \\
\hline Modèles Probabilistes & D. Békollé (FS) \\
\hline Méthodes Numériques, Analyse Numérique & I. Damakoa (FS) \\
\hline $\begin{array}{l}\text { Ateliers de Statistiques et } \\
\text { Analyse des Données }\end{array}$ & D. Tieudjo (ENSAI) \\
\hline Contrôle et Automatique & J.C. Kamgang (ENSAI) \\
\hline Génie Logiciel & Kolyang (FS) \\
\hline Réseaux, Systèmes Distribués & J.M. Nlong II (FS) \\
\hline Télédétection & André Ndi Nyoungui (IUT) \\
\hline
\end{tabular}

L'école camerounaise d'Epidémiologie Mathématique est allée au-delà des enseignements du professeur Gauthier Sallet par sa maittrise de la simulation informatique. Le Master d'Epidémiologie de l'Université de Ngaoundéré est un véritable master de Mathématiques Appliquées, dont les applications sont tangibles dans un contexte de pays en développement. L'Université de Yaoundé I emboîtera le pas à celle de Ngaoundéré l'année suivante (2009-2010).

\section{Le cycle de Doctorat/PhD à l'Université de Ngaoundéré}

L'Unité de Formation Doctorale "Mathématiques, Informatique et Applications (MIAP)" a été mise en place suite à une décision du 18 avril 2012 du Recteur de l'Université de Ngaoundéré. Le Master était déjà bien en place, la quatrième promotion était en cours de formation. A la rentrée de l'année académique 2012-2013, l’Unité de Formation Doctorale a accueilli ses premiers doctorants (Mathématiques et Informatique). Il était entendu 
qu'il fallait privilégier les cotutelles de thèses avec les universités étrangères (africaines, européennes,...) et les co-encadrements par des professeurs d'autres universités camerounaises et étrangères.

\section{Les premières thèses de Doctorat/PhD effectivement soutenues à 1'Université de Ngaoundéré}

Les trois premières thèses de Doctorat/PhD en Epidémiologie Mathématique ont été soutenues à l'Université de Ngaoundéré le 17 septembre 2017 pour les deux premières et le 18 septembre 2017 pour la troisième. Les soutenances se sont tenues dans le cadre de l'Atelier EpiMath VII, pour lequel l'Unité de Formation Doctorale bénéficia d'un financement du CETIC ${ }^{30}$. Le directeur scientifique et conférencier principal en fut Edward Lungu (Botswana International University of Science and Technology), incontestablement le plus grand professeur africain de Biomathématiques. Il présida avec autorité les jurys des deux premières thèses et éclaboussa de sa classe cet événement historique.

Voici une présentation des trois thèses, dans l'ordre de soutenance:

\section{KOUAKEP TCHAPTCHIE Yannick:}

"Stabilité et contrôle optimal de systèmes dynamiques en dimension infinie: Applications à l'Epidémiologie" (Directeurs: David Békollé, Arnaud Ducrot (Bordeaux), Duplex Elvis Houpa Danga). Dans son travail, le candidat procède en particulier à la modélisation de l'hépatite $\mathrm{B}$ et de l'impact de la vaccination contre cette maladie. Le modèle comporte six variables:

- Le temps (l'instant) t;

- L'âge chronologique (l'âge de l'individu);

- L'âge d'infection;

- Les trois variables spatiales $\mathrm{x}, \mathrm{y}$ et $\mathrm{z}$.
Le système est modélisé par un système d'équations aux dérivées partielles. Un résultat remarquable est le suivant: on peut remplacer la vaccination à trois doses (la première à l'instant 0 , la deuxième un mois après, la troisième un an après) par une vaccination à deux doses: la première à l'instant 0 , la deuxième à un instant qui est explicitement déterminé dans l'étude.

\section{FOTSA MBOGNE David Jaurès:}

"Contribution à la modélisation mathématique, au contrôle optimal et à l'observation de la dynamique de l'anthracnose de la baie du caféier" (Directeurs: David Békollé, Michel Ndoumbè Nkeng, Duplex Elvis Houpa Danga). Ce sujet d'épidémiologie végétale a été proposé par le Dr. Michel Ndoumbè Nkeng (IRAD) et il porte sur le contrôle optimal d'une maladie du caféier qui attaque les feuilles, puis les fruits, et provoque d'énormes pertes dans les récoltes: l'anthracnose de la baie du caféier (coffee berry disease). Le prix Ibni Oumar Mahamat Saleh des sociétés savantes françaises a été attribué à l'impétrant en 2014. Il lui a valu d'effectuer un séjour scientifique de quatre mois à l'Université d'Aix-Marseille auprès du professeur Etienne Pardoux. Son travail s'est alors enrichi de résultats de contrôle stochastique (ou aléatoire). Sous la supervision de Michel Ndoumbè Nkeng, l'impétrant travaille actuellement à l'élaboration d'un dispositif d'aide à la décision dans la lutte contre l'anthracnose.

\section{ABBOUBAKAR HAMADJAM:}

"Contributions à la modélisation mathématique et au contrôle de la dynamique des arboviroses: le cas de la dengue et du chikungunya" (Directeurs: Gauthier Sallet, Daniel Tieudjo, Jean Claude Kamgang). Les arboviroses sont des affections à caractère viral, transmises par des arthropodes hématophages. Elles présentent les mêmes symptômes que le paludisme ou la grippe. Les arbovirus sont des virus habituellement transmis,

${ }^{30}$ Centre d'Excellence des Technologies de l'Information et de la Communication: situé à l'Ecole Nationale Polytechnique de l'Université de Yaoundé I, il est financé par la Banque Mondiale. D’autres centres analogues fonctionnent dans d'autres pays africains (Sénégal, Bénin, Ghana...). 
dans les conditions naturelles, de vertébré à vertébré, par un arthropode hématophage, qui en constitue le vecteur.

Les arboviroses regroupent des maladies différentes quant à leur symptomatologie et surtout leur épidémiologie. Leur fréquence est réelle, ainsi que pour certaines leur gravité. Les arboviroses sont des maladies surtout tropicales. La modélisation mathématique de la dengue s'est imposée récemment en raison d'épidémies dans l'île française de la Réunion (2015, 2016, 2018). Le chikungunya est transmis par le moustique Aedes. Il survient dans la plupart des cas en Afrique (première épidémie connue: Tanzanie (1952)), en Asie et dans le sous-continent indien. Cependant, une épidémie de chikungunya a affecté en 2015 plusieurs pays des Amériques. La recherche sur les arboviroses est active, car contrairement au paludisme, elles ne sont pas des maladies des seuls pays pauvres.

Deux autres thèses de Doctorat/PhD seront soutenues à Ngaoundéré en 2019 par Ezekiel Dangbé (Directeurs: David Békollé et Irépran Damakoa) et Gabriel Kolaye Guilsou ("Contribution to theoretical assessment of the impact of climatic factors on the dynamical transmission of Cholera". Directeurs: David Békollé, Samuel Bowong Tsakou et Raymond Houé Ngouna (ENIT de Tarbes)). Ces travaux procurent une analyse scientifique nouvelle et profonde de l'épidémie meurtrière de choléra survenue en 2011 dans la région de l'ExtrêmeNord. Il faut signaler qu'Eric Brice Kokomo Ayissi soutiendra également à l'Université de Yaoundé I une thèse de Doctorat/PhD en Mathématiques sur la même maladie (avec applications à la même épidémie), dont l'analyse est réalisée avec des outils différents (équations aux dérivées partielles avec la prise en compte des variables spatiales, contrôle optimal avec la prise en compte des interventions chimiques, biologiques, et de la vaccination) (Directeur: Yves Sébastien Emvudu Wono).

Il faut enfin signaler que S.Y. Tchoumi soutiendra également en 2019 à l'Université de Ngaoundéré une thèse de Doctorat/PhD intitulée "Contribution à la modélisation et à la simulation des maladies à transmission vectorielle parasitaire" (Directeurs de thèse: Gauthier Sallet, Jean Claude Kamgang, Daniel Tieudjo). Les modèles proposés dans cette thèse se situent dans la lignée du modèle de Ross. Ils apportent une contribution aux stratégies d'éradication du paludisme qui visent la réduction de la capacité vectorielle. Dans ces contributions, on a une relaxation d'homogénéité dans laquelle la population des vecteurs est divisée en deux classes:

- la classe des vecteurs en quête de repas sanguin;

- la classe des vecteurs au repos.

Cette complexification tient compte, chez les vecteurs, du cycle extrinsèque d'incubation, dont l'auteur démontre une plus grande sensibilité de l'endémicité en comparaison avec les paramètres de contact usuels.

\section{Les thèses de Doctorat/PhD soutenues à l'Université de Yaoundé I, à l'Université de Buéa et à l'Université de Metz}

Cette liste suivante n'est pas exhaustive.

1. Djidjou Demassé Ramsès Emeric: "Modèles structurés et bifurcations dans les équations différentielles retardées à retard distribué". Soutenue à l'Université de Yaoundé I le 25 juin 2015. Directeurs: Marcel Dossa (FS), Jean Jules Tewa (ENSP), Samuel Bowong (Douala). Thème: Epidémiologie Mathématique.

2. Yatat Djeumen Ivric Valaire: ${ }^{31}$ "Analyse mathématique des modèles structurés en taille pour l'étude des interactions arbres-herbes dans les savanes". Soutenue à l'Université de Yaoundé I le 18 mai 2018. Directeurs: Jean Jules Tewa 
(ENSP), Yves Dumont (CIRAD ${ }^{32}$, Montpellier). Thème: Ecologie Mathématique.

3. Tchuinté Tamen Alexis: "Etude de quelques modèles d'interaction forêt-savanes: le cas du Cameroun". Soutenue à l'Université de Yaoundé I le 02 ao ut 2017. Directeurs: Samuel Bowong Tsakou (Douala), Yves Dumont (CIRAD, Montpellier), Pierre Couteron (IRD, Montpellier). Thème: Ecologie Mathématique.

4. Tchinda Mouofo Plaire: "Contrôle optimal et bifurcations dans les modèles épidémiologiques à retard distribué. Soutenue à l'Université de Yaoundé I le 30 mai 2016. Directeurs: Boulchard Mewoli (FS), Jean Jules Tewa (ENSP). Thème: Epidémiologie Mathématique.

5. Nkoa Onana Denis Fils: "Etude mathématique des interactions hôtes pathogènes et émergence des blips lors du traitement par la méthode HAART et commande optimale de l'infection par le VIH'. Soutenue à l'Université de Yaoundé I le 09 janvier 2015. Directeurs: David Békollé, Boulchard Mewoli (FS). Thème: Epidémiologie Mathématique HIV.

6. Bongor Danhree: "Contrôle Optimal dans les Equations Différentielles Stochastiques et Applications en Epidémiologie". Soutenue à l'Université de Yaoundé I le 22 juin 2018. Directeurs: Yves Sébastien Emvudu Wono (FS), KoÏna Rodoumta (N'Djamena). Thème: Epidémiologie Mathématique, Probabilités, Contrôle Optimal.

7. Mary Nforba sp Fomboh: "Studies on novel approaches in modelling malaria transmission dynamics". Soutenue à l'Université de Buéa en 2017. Supervisor: Gideon Akumah Ngwa. Thème: Biomathematics.

\section{Woldegebriel Assefa Woldegerima:} "Mathematical modeling of the immunopathogenesis of the within human host and within vector-host parasites". Soutenue à l'Université de Buéa le 18 juin 2018. Supervisors:
Gideon Akumah Ngwa, Miranda TebohEwungken (Lehigh University, USA). Thème: Biomathematics.

9. Léontine Nkague Nkamba: "Robustesse des seuils en épidémiologie et stabilité asymptotique d'un modèle à infectivité et susceptibilité différentielle". Soutenue le 23 novembre 2012 (Université de Metz et Université Gaston Berger, Saint-Louis (Sénégal)). Directeur: G. Sallet. Thème: Epidémiologie Mathématique.

\section{Conclusion: L'urgence de cadres de collaboration entre mathématiciens,} biologistes et professeurs de médecine

Il est maintenant urgent d'instaurer des cadres de collaboration entre mathématiciens, biologistes et professeurs de médecine. La recherche moderne en biologie et en médecine s'appuie sur les modèles, les lois de la bioéthique l'y contraignent! D’emblée, les mathématiciens proposent le projet conjoint suivant: fabriquer un nouveau vaccin à deux doses contre l'hépatite B. Le gouvernement camerounais, par l'intermédiaire du ministère de la Santé Publique, du ministère de l'Enseignement Supérieur et du ministère de la Recherche Scientifique, doit instituer des projets de collaboration y relatifs avec l'OMS, l'Institut Pasteur, l'IRD, l'OCEAC, les industries pharmaceutiques. La recherche sur le paludisme, notre "maladie sociale", est complexe et elle manque de financements parce que c'est une maladie de pays pauvres. Elle relève de la responsabilité de nos pays, de même que la recherche sur les autres maladies tropicales négligées. Mais cela n'exclut pas d'apporter aussi notre contribution à la lutte contre le VIH. Il convient de respecter la règle, imiter les bons exemples et les surpasser: le Nigéria avec le Projet Garki, le Sénégal avec le Projet Dielmo-Ndiop.

Un cadre de collaboration a été réalisé par le DFG (Deutsche Forschungsgemeinschaft), organisme

${ }^{31}$ Prix Ibni Oumar Mahamat Saleh 2015. Le Cameroun remporte le prix pour la deuxième année consécutive.

${ }^{32}$ Centre de Coopération Internationale en Recherche Agronomique pour le Développement. 
allemand de coopération scientifique, en coopération avec AIMS (African Institute for Mathematical Sciences). Dans ce cadre, un atelier s'est tenu à Douala du 20 au 25 février 2017 sur le thème "Mathematics against Malaria: A Holistic Approach". Cet atelier a réuni des mathématiciens, biostatisticiens et bioinformaticiens allemands et camerounais (parmi lesquels Martin Eichner, Marcel Schulz, Bernhard Renard, Gideon Akumah Ngwa, Samuel Bowong, Jean Jules Tewa et l'auteur de cet article) autour du professeur camerounais de biologie, Wilfred Fon Mbacham. Avaient également participé à cet atelier DFG-AIMS trois mathématiciens de la diaspora: Prof. Miranda Teboh-Ewungkem (Lehigh University, Bethlehem, Pennsylvania, USA), Dr. Wilfred Ndifon (AIMS, Cape Town, South Africa), Dr. Calistus Ngonghala (College of Liberal Arts and Sciences, University of Florida, Gainesville, Florida, USA) ${ }^{33}$. L'objectif à terme est de mutualiser les outils de modélisation issus des mathématiques, de l'informatique, des biostatistiques et de la physique pour élaborer des stratégies d'éradication du paludisme dans le monde.

Nous avons appris à cette occasion par Thomas Dan Otto (Wellcome Trust Sanger Institute ${ }^{34}$, Cambridge, Grande- Bretagne) que sur le plan de la génétique, le Plasmodium Falcifarum n'était encore qu'à une étape préliminaire de son développement, il est dans sa 'prime jeunesse'. Cela indique que la recherche sur le vaccin contre le paludisme en zone endémique est encore plus passionnante que prévu. Sans doute, Wilfred Mbacham a perçu les enjeux de cette rencontre, qui allait bien dans le sens de ses intérêts scientifiques. Pour la petite histoire, une délégation de mathématiciens, conduite par Gideon Akumah Ngwa et Martin Eichner, l'a ensuite suivi dans son laboratoire de Yaoundé pour examiner ses données ${ }^{35}$.

\section{Les perspectives}

Il y a du grain à moudre, du pain sur la planche. Pendant l'épidémie de fièvre Ebola qui a sévi en Afrique de l'Ouest de 2013 à 2016, l'OMS a déploré que les modèles proposés par les mathématiciens n'aient pas été très utiles pour contrôler l'épidémie. Pendant son séjour à Ngaoundéré en septembre 2017, Edward Lungu a témoigné qu'il avait lui-même proposé un modèle qui n'avait pas été validé lors de sa confrontation avec les données sur le terrain. En réponse à cette interrogation, Samuel Bowong Tsakou a rétorqué que les modèles existants ne prenaient pas en compte les réservoirs de virus (les grands singes, l'environnement,...) et qu'il venait de proposer en sujet de thèse d'élaborer de nouveaux modèles ainsi corrigés. Le travail de thèse est en cours...

13. Annexe 1: La liste des chercheurs seniors permanents en Epidémiologie Mathématique

1. Samuel Bowong Tsakou, Maître de Conférences, Faculté des Sciences, Université de Douala. Spécialité: Contrôle et Automatique, Epidémiologie et Ecologie.

2. Jean Jules Tewa, Maitre de Conférences, Ecole Nationale Supérieure Polytechnique, Université de Yaoundé I. Spécialité: Contrôle et Automatique, Epidémiologie et Ecologie.

3. Gideon Akumah Ngwa, Maître de Conférences, Faculty of Science, University of Buea. Spécialité: Biomathematics.

4. Yves Sébastien Emvudu Wono, Maitre de Conférences, Faculté des Sciences, Université de

\footnotetext{
${ }^{33}$ Le professeur Teboh-Ewungkem et le Dr. Ngonghala ont obtenu leur Master à l'Université de Buéa (directeur: Gideon Akumah Ngwa)

${ }^{34}$ Les ordinateurs utilisés dans cet institut pour étudier le génome du P. falcifarum coûtent quelques milliards de FCFA l'unité.

${ }^{35}$ En novembre 2018, l'ambassadeur des Etats-Unis a effectué une visite à l'Université de Ngaoundéré. Lors d'une cérémonie de remise de livres, un collègue informaticien lui a demandé l'aide de son pays pour faire émerger au Cameroun des personnalités comme Bill Gates et Steve Jobs (le fondateur de la firme Apple). L'ambassadeur a répondu qu'"il est du devoir du gouvernement camerounais de créer un environnement propice pour qu'après avoir échoué à plusieurs reprises, un innovateur puisse enfin réussir de la plus belle des façons”.
} 
Yaoundé I. Spécialité: Problèmes inverses et bien posés, Calcul Scientifique, Epidémiologie.

5. Daniel Tieudjo, Maître de Conférences, Ecole Nationale Supérieure des Sciences AgroIndustrielles, Université de Ngaoundéré \& AIMS Cameroon. Spécialité: Algèbre, Cryptographie, Statistiques, Epidémiologie.

6. Jean Claude Kamgang, Maître de Conférences, Ecole Nationale Supérieure des Sciences Agro-Industrielles, Université de Ngaoundéré. Spécialité: Contrôle et Automatique, Epidémiologie.

7. Duplex Elvis Houpa Danga, Maitre de Conférences, Faculté des Sciences, Université de Ngaoundéré. Spécialité: Equations aux Dérivées Partielles, Systèmes Dynamiques en dimension infinie, Epidémiologie.

8. Irépran Damakoa, Maître de Conférences, Faculté des Sciences, Université de Ngaoundéré. Spécialité: Analyse Numérique, Epidémiologie.

9. Berge Tsanou, Maître de Conférences, Faculté des Sciences, Université de Dschang. Spécialité: Epidémiologie.

10. Michel Ndoumbè Nkeng, Maître de Recherches, Institut de Recherche Agronomique pour le Développement. Spécialité: Agronomie, Statistiques et Analyse de Données.

11. William Shu, Chargé de Cours, Faculty of Science, University of Buea. Spécialité: Biomathematics, Computer Science.

12. Joseph Mbang, Chargé de Cours, Faculté des Sciences, Université de Yaoundé I. Spécialité: Epidémiologie.

13. Léontine Nkagué Nkamba, Chargée de Cours, Ecole Normale Supérieure, Université de Yaoundé I. Spécialité: Epidémiologie.

14. Patrice Soh Takam, Chargé de Cours, Faculté des Sciences, Université de Yaoundé I. Spécialité: Statistiques et Analyse des Données, Modélisation stochastique.

15. Mary Fomboh, Chargée de Cours, Faculty of Science, University of Buea. Spécialité: Biomathematics.
16. Vivient Corneille Kamla, Chargé de Cours, Ecole Nationale Supérieure des Sciences AgroIndustrielles, Université de Ngaoundéré. Spécialité: Informatique, Epidémiologie (modélisation individu-centrée, simulation à base d'agents).

17. Jean Luc Dimi, Maître de Conférences, Faculté des Sciences, Université Marien Ngouabi, Brazzaville. Spécialité: Statistiques, Epidémiologie, Modélisation stochastique.

18. Koïna Rodoumta, Maître de Conférences, Faculté des Sciences Exactes et Appliquées, Université de N’Djamena. Spécialité: Epidémiologie, Probabilités, Contrôle et Automatique.

\section{Annexe 2: Un florilège de publications.}

L'équipe d'Afrique Centrale d'Epidémiologie Mathématique a son actif plus de 70 publications scientifiques. Certaines ont déjà été citées dans le texte. Le choix ci-dessous de 10 publications relève de la seule responsabilité de l'auteur des présentes lignes.

1. J.C. Kamgang, G. Sallet (2008). "Computation of threshold conditions for epidemiological models and global stability of the disease-free equilibrium (DFE)". Mathematical Biosciences 213, 1-12.

2. P. Auger, E. Kouokam, G. Sallet, M. Tchuenté, B. Tsanou (2008). "The Ross-Macdonald model in a patchy environment". Math. Biosci. 216, no. 2, 123-131.

3. D.J. Fotsa Mbogné, D.E. Houpa Danga, D. Békollé, M. Ndoumbè Nkeng, C. Thron (2014). "Mathematical modelling and optimal control of anthracnose". BIO-MATH. 3, no. 1, 1404161, 16 pp.

4. B. Danhree, Y.S. Emvudu Wono, K. Rodoumta (2017). "Optimal control of the treatment frequency in a stochastic model of tuberculosis". BIOMATH. 6, no. 1, 1705077, 17 pp.

5. J.J. Tewa, J.L. Dimi, S. Bowong (2009).

"Lyapounov functions for a dengue disease 
transmission model". Chaos Solitons Fractals 39, no. 2, 936-941.

6. E. Dangbé, D. Békollé, I. Damakoa, A. Perasso (2017). "Impact of Hygiene, Famine and Environment on Transmission and Spread of Cholera". Math. Model. Nat. Phenom. 12 (2), 4-21. 7. V. Yatat, P. Couteron, J.J. Tewa, S. Bowong, Y. Dumont (2017). "An impulsive modelling framework of fire occurrence in a size structured model of tree-grass interactions for savanna ecosystems". J. Math. Biol. 74, no. 6, 1425-1482. 8. Y. Kouakep Tchaptchié, A. Ducrot, D.E. Houpa Danga (2013). "A model for hepatitis B with chronological and infection ages". Appl. Math. Sci. (Ruse) 7, no. 117-120, 5977-5993.

9. M. L. Mann Manyombè, B. Tsanou, J. Mbang, S. Bowong (2017)."A metapopulation model for the population dynamics of anopheles mosquito". Appl. Math. Comput. 307, 71-91.

10. R. Houé, M.A. Aziz-Alaoui, G. Kolaye, S. Bowong, M. Cadivel (2019). "Mathematical assessment of the role of environmental factors on the dynamical transmission of cholera". Communications in Nonlinear Science and Numerical Simulation 67, 203-222.

15. Annexe 3: Les soutiens obtenus au long du parcours Cet annexe répertorie les soutiens obtenus pour réaliser les différentes activités de mise en place du thème de recherche "Epidémiologie Mathématique" en Afrique Centrale. C'est également le lieu de renouveler les remerciements aux organismes donateurs.

Activité 1: Séminaire de l'Union Mathématique Africaine (UMA), Département de Mathématiques, Faculté des Sciences, Université de Yaoundé I, 1997.

-Union Mathématique Africaine (UMA);

-Centre International de Mathématiques Pures et Appliquées (CIMPA), Nice (France);

-Département de Mathématiques, Faculté des

Sciences, Université de Yaoundé I.

${ }^{36}$ Projet financé par le ministère français des Affaires Etrangères (2004-2008).

${ }^{37}$ Institut des Hautes Etudes Scientifiques, Bures-sur-Yvette (France).
Activité 2: Célébration de l'Année Mathématique Mondiale, Ministère de la Recherche Scientifique et Technique, Yaoundé, aout-septembre 2000.

-Ministère de la Recherche Scientifique et Technique, Yaoundé;

-Ministère de l'Enseignement Supérieur, Yaoundé; -Centre International de Mathématiques Pures et Appliquées (CIMPA), Nice (France);

-International Centre for Theoretical Physics (ICTP), Trieste (Italie);

-UNESCO;

-Union Mathématique Internationale (UMI);

-Institut National (français) de la Recherche Agronomique (INRA);

-Institut National (français) de la Recherche en Informatique et Automatique (INRIA).

Activité 3: Ateliers "Mathématiques et Malaria", AUF, Yaoundé, 2003, Université Marien Ngouabi, Brazzaville, 2004.

-Agence Universitaire de la Francophonie (AUF), Projet PAS 01;

-Université de Yaoundé I;

-Université Marien Ngouabi, Brazzaville. Activité

4: Atelier EpiMath I de Yaoundé, 7-11 novembre 2005.

-Institut (français) de Recherche pour le Développement, Bondy (France);

-Projet SARIMA (Soutien aux Activités de Recherche en Informatique et Mathématiques en Afrique ${ }^{36}$;

-Fondation Schlumberger, Programme IHES ${ }^{37}$ Schlumberger;

-Institut de Médecine Tropicale du Service de Santé des Armées, Marseille (France);

-Institut National (français) de la Recherche en Informatique et Automatique (INRIA);

-Université des Sciences et Techniques de Masuku, Franceville (Gabon). 
Activité 5: Ateliers EpiMath II, IV et VI de Brazzaville, mars 2007, septembre 2009 et mai 2014. -Projet SARIMA (Soutien aux Activités de Recherche en Informatique et Mathématiques en Afrique);

-Université Marien Ngouabi, Brazzaville;

-International Science Programme (ISP) ${ }^{38}$, Uppsala (Suède);

-Fondation Schlumberger, Programme IHESSchlumberger;

-Université Marien Ngouabi, Brazzaville;

-Délégation Générale de la Recherche Scientifique et Technique, Brazzaville.

Activité 6: Ateliers EpiMath III, V et VII de Ngaoundéré, 2008, 2012 et 2017.

-Agence Universitaire de la Francophonie (AUF), Projet MIME;

-Institut de Médecine Tropicale du Service de Santé des Armées, Marseille (France);

-Centre International de Mathématiques Pures et Appliquées (CIMPA), Nice (France);

-Fondation Schlumberger, Programme IHESSchlumberger;

-Université de Ngaoundéré, Rectorat;

-Faculté des Sciences, Université de Ngaoundéré;

-Ecole Nationale Supérieure des Sciences Agro-

Alimentaires et Industrielles, Université de Ngaoundéré;

-Institut Universitaire de Technologie, Université de Ngaoundéré.

Activité 7: Mini-GIRAGA ${ }^{39}$, Université de Yaoundé I, janvier 2015. Conférenciers:

Khalil Eqzinbi (Marrakech), Arnaud Ducrot (Bordeaux).

-Centre International de Mathématiques Pures et Appliquées (CIMPA), Nice (France);

-Union Mathématique Internationale (UMI);
-Association pour la Promotion de la Science en Afrique (APSA), Paris (France).

Activité 8: Mobilités de thèses et rencontres de travail, 2004-2008.

-Projet SARIMA (Soutien aux Activités de Recherche en Informatique etMathématiques en Afrique).

16. Annexe 4: Signification des abbréviations AIMS: African Institute for Mathematical Sciences APSA: Association pour la Promotion de la Science en Afrique

AUF: Agence Universitaire de la Francophonie CETIC: Centre d'Excellence des Technologies de l'Information et de la Communication CIMPA: Centre International des Mathématiques Pures et Appliquées

CIRAD: Centre de Coopération Internationale en Recherche Agronomique pour le Développement DEA: Diplôme d'Etudes Approfondies

ENSAI: Ecole Nationale Supérieure des Sciences Agro-Alimentaires et Industrielles

ENSP: Ecole Nationale Supérieure Polytechnique, Université de Yaoundé I

EpiMath: Réseau d'Afrique Centrale de Modélisation Informatique et Mathématique en Epidémiologie et en Immunologie

FS: Faculté des Sciences

GIRAGA: Groupe Interafricain de Recherche en Analyse, Géométrie et Applications

ICTP: International Centre for Theoretical Physics IMSP: Institut de Mathématiques et Sciences Physiques de Porto Novo (Bénin)

INRA: Institut National (français) de la Recherche Agronomique

INRIA: Institut National (français) de la Recherche en Informatique et Automatique

\footnotetext{
${ }^{38}$ Projet "Analysis, Geometry and Applications" in the Cameroonian universities (2003-2008).

${ }^{39}$ GIRAGA: Groupe Interafricain de Recherche en Analyse, Géométrie et Applications. Ce groupe organise tous les deux ans depuis 1986, les séminaires GIRAGA, alternativement à Yaoundé et au Bénin. La coordination en est assurée par les départements de Mathématiques des facultés des Sciences de l'Université de Yaoundé I et de l'Université d'Abomey-Calavi, et l'IMSP.
} 
IRAD: Institut de Recherche Agronomique pour le Développement

IRD: Institut (français) de Recherche pour le Développement

ISP: International Science Programme

IUT: Institut Universitaire de Technologie

MIME: Projet "Modélisation Mathématique et

Informatique en Epidémiologie"

OMS: Organisation Mondiale de la Santé

SARIMA (Soutien aux Activités de Recherche en Informatique et Mathématiques en Afrique)

UE: Unité d'Enseignement

UYI: Université de Yaoundé I.

\section{Remerciements}

L'auteur remercie les rapporteurs pour leur lecture attentive du manuscrit et les suggestions utiles qu'ils (elles) ont formulées. Il exprime sa gratitude à Gideon Akumah Ngwa, Yves Emvudu, Irépran Damakoa et Joseph Mbang, qui ont contribué par des corrections et des informations complémentaires à l'amélioration de la qualité de cet article.

\section{References}

[1] Dutertre, J. (1976). "Etude d'un modèle épidémiologique appliqué au paludisme". Ann. Soc. Belge Méd. Trop. 56 (3), 127-141.
[2] Dutertre, J. (2001). "Mathématiques et développement en Afrique”. La Jaune et la Rouge, Revue mensuelle de la Société amicale des anciens élèves de l'Ecole Polytechnique 565, 39-41.

[3] Kamgang, J.C., V.C. Kamla, S.Y. Tchoumi (2014). "Modeling the Dynamics of Malaria Transmission with Bed Net Perspective". Applied Mathematics 5, 3156-3205. http://dx.doi.org/ 10.4236/am.2014.519298.

[4] Ngwa, G.A., W. Shu (2000). "A mathematical model for endemic malaria with variable human and mosquito populations". Mathematical and Computer Modelling 32, 747-763.

[5] Ross, R. (1911). "The prevention of malaria”. John Murray, London. 